\title{
MODELO DIAGNÓSTICO DE RESPONSABILIDAD SOCIAL EMPRESARIAL PARA MIPYMES DEL SECTOR DE RECICLAJE DEL BARRIO TOBERÍN EN LA CIUDAD DE BOGOTÁ ${ }^{*}$
}

\section{DIAGNOSTIC MODEL OF CORPORATE SOCIAL RESPONSIBILITY FOR SMES IN THE RECYCLING SECTOR AT TOBERÍN NEIGHBORHOOD IN BOGOTA CITY}

Recibido: 8 de enero de 2020

Evaluado: 15 de febrero de 2020

Aprobado: 6 de mayo de 2020

Cómo citar este artículo: García Vargas, A. M., Paredes Corredor, E. A., Figueroa Camacho. L. V. y Marin Chaves, Y. V. (2020). Modelo diagnóstico de responsabilidad social empresarial para mipymes del sector de reciclaje del barrio Toberín en la ciudad de Bogotá. Revista Estrategia Organizacional, 9(2). doi: 10.22490/25392786.4042

\author{
Adriana Milena García Vargas* \\ Universidad de San Buenaventura \\ Orcid: https://orcid.org/0000-0002-1523-9466 \\ Edgar Andrés Paredes Corredor ${ }^{* \star}$ \\ Universidad de San Buenaventura \\ Orcid: https://orcid.org/0000-0002-4173-994X \\ Laura Viviana Figueroa Camacho ${ }^{* \star *}$ \\ Universidad de San Buenaventura \\ Orcid: https://orcid.org/0000-0003-0613-7457 \\ Yeimy Viviana Marin Chaves ${ }^{\star \star \star \star *}$ \\ Universidad de San Buenaventura \\ Orcid: https://orcid.org/0000-0002-1142-3370
}

\footnotetext{
* $\quad$ Artículo de investigación resultado del proyecto realizado en la Universidad San Buenaventura.

** Administradora de Empresas de la Universidad de San Buenaventura. Correo electrónico: amgarciav@academia. usbbog.edu.co

*** Administrador de Empresas de la Universidad de San Buenaventura. Investigador del Semillero de Bioconomía. Correo electrónico: eaparedesc@academia.usbbog.edu.co

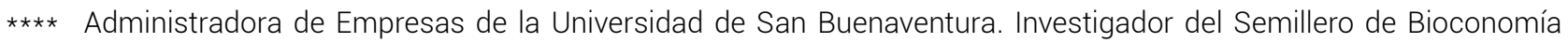
Correo electrónico: Ivfigueroa@academia.usbbog.edu.co

***** Administradora de Empresas de la Universidad de San Buenaventura Correo electrónico: yemarin@usbbog.edu.co
} 


\section{RESUMEN}

Introducción: Se realizó una revisión de antecedentes sobre la importancia que ha tomado en los últimos años la Responsabilidad Social Empresarial (RSE), su fuerte aporte al Desarrollo Sostenible y el impacto que esta tiene en los stakeholders de las compañías. Metodología: se lleva a cabo una investigación con la finalidad de obtener un modelo Diagnóstico frente a la RSE para ser aplicado a las mipymes del sector de reciclaje del Barrio Toberín en Bogotá. El modelo diagnóstico permitirá en una segunda fase del proyecto medir el porcentaje de cumplimiento de estas empresas frente al factor de RSE e indicar posibles prácticas nuevas que podrían desarrollar. El objetivo es construir una herramienta de diagnóstico con base en la situación actual de las mipymes del sector de reciclaje, de fácil manejo y comprensión para los miembros de estas organizaciones, la cual les servirá de insumo para la implementación de iniciativas claras de RSE que estén a su alcance y acorde a su contexto. Resultados: Al lograr alinear sus procesos actuales apuntando hacia una RSE que potencialice las prácticas existentes y les proporcione claridad sobre nuevas rutas a seguir, se considera que lograrán también impactar positivamente en el Desarrollo Sostenible, no solo de sus propias empresas, sino también en el sector económico al que pertenecen y en la comunidad en general en la que desarrollan sus actividades. Conclusiones: lo anterior, les ayudará a fortalecer la confianza de sus proveedores hacia ellos, su imagen frente a los clientes, aumentar las posibilidades de generar nuevos ingresos o inversiones, entre muchos otros beneficios que la RSE y el Desarrollo Sostenible traen consigo.

Palabras clave: Stakeholders, Responsabilidad Social Empresarial (RSE), reciclaje y desarrollo sostenible.

\section{ABSTRACT}

Introduction: It was made a background check about the increasing of importance that had had the Social Responsibility term (SR) in the last years due to its contribution to Sustainable Development and its impact on stakeholders. Methodology: the current investigation analyze the conscious and unconscious performances of the SMEs in the recycle sector at Toberín neighborhood in Bogotá. Results: Afterwards, A diagnostic model is developed in the second part of this investigation to evaluate the accomplishment level of SR factor in the companies and it is mentioned new performances that could be set up by them. The objective is to create an easy and comprehensive diagnostic tool base on SMEs' current situation in the recycle sector. Moreover, it guides the organization members to set up the initiatives about SR which are within the reach of them and according to their context. It is considered that the companies impact 
positively on Sustainable Development, not only in its companies, but also in the economic sector and the community in general where they carry out. This happen when they align their current performances toward SR which potentialize the actual executions and give them clarity about new routes to follow. Conclusions: Furthermore, it also helps them to strengthen the suppliers' trust, to get a better image as company to its customers, to raise its possibilities to generate new incomings or investments, among many others SR and Sustainable Development's benefits.

Key words: Stakeholders, SMEs, Social Responsibility, Strategy and Sustainable Development

\section{INTRODUCCIÓN}

Durante los últimos años, la Responsabilidad Social Empresarial (RSE) ha tomado mayor fuerza e importancia en el mercado a nivel mundial, por lo cual las empresas multinacionales se han visto avocadas a involucrar la RSE en sus procesos, también motivadas por los beneficios y mejoras que esto trae para la organización en general (Vives, 2014). Es así como se han empezado a incluir en la misión y visión de las empresas elementos de RSE como el medio ambiente, la transparencia, la comunidad y los valores; dichos elementos se instauran desde la raíz de las instituciones y desde allí germinan llegando a impregnar en todos los proyectos, actividades y procesos que las empresas desarrollan.

En América Latina no han pasado desapercibido estos cambios. Con el fin de obtener los beneficios que la RSE trae a la sociedad y a la empresa, como lo son el aumento del crecimiento y desarrollo económico, mejor imagen en el mercado para la empresa y mayores utilidades; las organizaciones latinoamericanas han empezado a adoptar las directrices que la RSE aporta. Se han llevado a cabo investigaciones que han permitido identificar las prácticas que se deben implementar y las herramientas a utilizar con el fin de lograr que la RSE haga parte esencial de las organizaciones y permita así a las empresas mejorar sus procesos mientras aportan positivamente a la sociedad que los rodea (Playán, 2010).

En Colombia, la responsabilidad social empresarial ha sido un tema de discusión y complejidad para la mipyme, pues su relación con los estándares globales, los han puesto a pensar como 
realmente ser empresas que se incorporen día a día a esta tendencia (Pastrana, 2014). Sin embargo, este trabajo pretende diagnosticar la gestión de las mipyme del sector de reciclaje del barrio Toberín en Bogotá. Es por esto que para dar una estructura sólida al presente documento se realizará un diagnóstico que permita conocer las aplicaciones concretas de la RSE en estas empresas del Sector y cómo mediante un modelo estándar podemos encaminarlas a un diseño general estratégico de pasos para desarrollarlo de manera correcta. Por esto, los métodos y el desarrollo de la investigación se presentan a continuación.

\section{CONTENIDO}

Durante la primera etapa de esta investigación se busca indagar sobre el concepto de Responsabilidad Social Empresarial a través de una revisión bibliográfica de trabajos, libros e investigaciones desarrolladas en las últimas décadas que permitan fortalecer los conocimientos y preparar a los investigadores para el desarrollo de la segunda etapa que consiste en la creación de un modelo diagnóstico.

Dado que la mirada central de esta investigación está puesta sobre la Responsabilidad Social Empresarial, se realiza un análisis de la evolución de este término a lo largo de la historia. En principio, se encuentra a Adam Smith quien escribió la "Teoría de los Sentimientos Morales", en donde se habla de la ética aplicada en todos los procesos de una empresa y de cómo los seres humanos pueden tener un proceso de simpatía o empatía (Smith, 2013). En otras palabras, la persona es capaz de ponerse en los zapatos del otro, aun cuando no obtenga ningún beneficio pro hacerlo.

Como continuidad a estos principios éticos nace el termino de Responsabilidad Social Empresarial, definida por la OIT (Organización Internacional del Trabajo) como una actitud consciente y responsable de las mismas organizaciones hacia el bienestar común. Esta organización no solo unió a diferentes líderes de países, sino que también dio una vista general de cuáles eran las variables importantes en el trabajo decente del mundo.

Por otro lado, en 1953, Howard Bowen acuña formalmente el término de Responsabilidad Social a través de su obra "Responsabilidades Sociales del Hombre de Negocios". Esta obra muestra la primera definición de este término indicando las obligaciones de los hombres de negocios al aplicar políticas empresariales, tomar decisiones o seguir líneas de acción que son deseables 
en términos de objetivos y valores de nuestra sociedad (Bowen, 1953). Por lo anterior Bowen se denominó el padre de la RSE.

También se encuentran los autores Porter y Kremer quienes fortalecen las teorías instrumentales que plantean como las actividades sociales llegan a generar el cumplimiento de los objetivos que buscan una mayor riqueza empresarial. La presente investigación se fundamente fuertemente en estas teorías considerando. En sus obras "The Link Between Competitive Advantage and Corporate Social Responsibility" "y "The Competitive Advantage of Corporate Philantrophy" los autores afirman que se pueden obtener retornos positivos para la organización a través de prácticas filantrópicas proyectadas a mediano y largo plazo. Aun así, algunos inversionistas exigen aumento en los beneficios a corto plazo generando una difícil disyuntiva para los directivos de la empresa.

Con base en lo anterior, Porter y Kramer plantean que las prácticas de RSE hacen parte esencial del plan de posicionamiento estratégico de la empresa generando valores compartidos con los stakeholders. Lo anterior se puede ver como una ventaja competitiva de las organizaciones frente su competencia, la cual construye una relación sólida con los grupos de interés que se transforma a su vez en una "red de relaciones de confianza" (del Castillo, 2008)

Finalmente, apoyando la temática de la responsabilidad social empresarial, Edward Freeman, definió a los Stakeholder quienes son impactados por la responsabilidad social empresarial de forma directa. Con el paso de los años Freeman se ha encargado de moldear y mejorar dicha definición llegando a la siguiente: "cualquier grupo o individuo que puede afectar o ser afectado por el logro de los objetivos empresariales" (Freeman, 2008).

\section{METODOLOGÍA}

\section{DISEÑO METODOLÓGICO}

La presente investigación es de tipo cuantitativo, ya que se realizará un análisis de los resultados obtenidos luego de aplicar encuestas elaboradas con preguntas estructuradas a los miembros de las mipyme del sector de reciclaje en el barrio Toberín de Bogotá. Con esto se pretende identificar que entienden por RSE y constatar si las problemáticas frente a la implementación de este tema están relacionadas con la falta de conocimientos sobre el mismo. Adicionalmente, se pretende 
conocer las prácticas de RSE que llevan a cabo en las empresas por iniciativa propia y también aquellas que desarrollan sin ser conscientes de que impactan en la RSE.

Se pretenden analizar variables referentes a la protección de los Derechos Humanos, el cumplimiento de los derechos laborales de los colaboradores, la protección del medio ambiente y los procedimientos con los que cuentan para evitar los actos de corrupción dentro de la organización. Con base en los resultados, se generará el modelo diagnóstico que pretenderá abarcar estos mismos temas para así presentar un escenario claro cumplimiento o no de Responsabilidad Social Empresarial.

\section{MÉTODO DE INVESTIGACIÓN}

Este trabajo se desarrolla utilizando un método inductivo, debido a que se sacan conclusiones por medio de la observación a las empresas del sector con el fin de conocer las buenas prácticas y aplicación de la Responsabilidad Social Empresarial, para lo cual se seguirán los pasos de observación, formulación de hipótesis, verificación y tesis. (Newman, 2006)

\section{TIPO DE ESTUDIO}

Además, resulta ser de carácter descriptivo, puesto que es la utilizada para describir la realidad de situaciones, eventos, personas o grupos que se pretenden analizar. En este caso se describirán las situaciones identificadas en aquellas mipyme del Barrio Toberín en la ciudad de Bogotá, por medio de las etapas de examinar las características del tema a investigar, definirlo y formular la hipótesis. (Paneque, 1998)

\section{RESULTADOS}

En la primera etapa de la investigación, se consultaron antecedentes, los cuales se enmarcaron en tres grandes apartados. En el primero se hace referencia a las definiciones conceptuales; temas como lo son la Responsabilidad Social Empresarial como ventaja competitiva, el nuevo enfoque de la RSE y el nacimiento de la teoría que habla de la existencia de una responsabilidad de la empresa hacia la sociedad y las personas que están en ella, esta responsabilidad social entendida como una responsabilidad integral, esto es, económica y social. (Reyno Momberg, 2007). 
También se descubrieron algunos modelos con los cuales se puede evaluar la Responsabilidad social en las empresas, por consiguiente se realizó una identificación y revisión de diferentes teorías instrumentales y humanísticas que fueron fundamentales en la investigación para la interpretación de unos modelos determinados y su función dentro de la compañía, para llegar a un resultado final realizaron una comparación entre los mismos para llegar a identificar no solo para qué sirve el modelo sino también, cuáles son sus diferencias, entre otras características que dan a conocer dicha herramienta. (Plasencia Soler, Marrera Delgado, Bajo Sanjuán, y Nicado García, 2018).

Por otro lado, la teoría de Chiavenato hace enfrentar el concepto de RSE con la historia de manera que conceptualiza su teoría como una "obligación gerencial que una organización asume para tomar acciones que protegen y mejoran el bienestar de la sociedad y los intereses organizacionales específicamente" (Chiavenato, 2006)

Además es claro y preciso afirmar que la Responsabilidad Social Empresarial, a pesar de, que el concepto de RSE ha ido ganado varios hitos de importancia ( como ser un centralizador de mejora continua en todas las organizaciones), es un concepto que no debe contentar al gestor o administrador de la compañía, con el simple hecho de velar por intereses corporativos regidos en lo legal, sino ir más allá dejando fluir de manera continua la ética que se aborda desde la RSE siendo esencial las acciones que se generan en los entornos ambiental y social. En consecuencia, la Responsabilidad social se concentra en ser una "exigencia ética" y una estrategia corporativa y estratégica para el correcto desarrollo de las empresas Mipyme que en la actualidad desarrollan un valor agregado para estas mismas. (Guerra, 2007)

En el segundo apartado se encuentran los Beneficios Empresariales, Actividades y Casos, donde se revisaron investigaciones sobre varios tipos de casos empresariales e igualmente se indagó en otros componentes como el componente jurídico que puede soportar la implementación de un sistema de Responsabilidad Social Empresarial. Acevedo Guerrero y su equipo van más allá de las 4 áreas fundamentales que normalmente se involucran en este ámbito, y dan una mirada micro y macro del área jurídica fortaleciendo el concepto de RSE con análisis cualitativo y legal y enmarcado en un espacio, no solo global sino especifico en Colombia. Se obtuvo como resultado un estudio bastante estructurado y rígido de lo que puede afectar un contexto de solo teorías y 
no prácticas. Es así como podemos ultimar que la RSE a medida que se implementa en Colombia, carece de practicidad y se conceptualiza de manera que las empresas solo enfocan su mirada en lo técnico y el "papel" que las certifica y no van más allá de las repercusiones prácticas que pueden apoyar cien por ciento el dinamismo de la implementación. (Acevedo Guerrero, Zárate Rueda, y Garzón Ruiz, 2013).

A continuación, se muestra como en una empresa no solo evalúan la responsabilidad social empresarial también evalúan la lealtad de clientes debido a que según uno de los estudios que referencia el artículo, concluyeron que la responsabilidad social empresarial impacta directamente la lealtad del cliente y la valoración que estos le dan al servicio (Pérez Ruiz, García de los Salmones Sánchez y Rodríguez del Bosque 2008). El articulo muestra que tomaron distintas variables (Variable independiente "RSE" compuesta por 3 dimensiones "ético-legal, filantrópica y valoración global" Variable dependiente "Lealtad" compuesta por 4 dimensiones "Actitudinal cognitiva, actitudinal afectiva, actitudinal conativa y comportamental") las cuales alimentaron el instrumento que tenía como finalidad medir la RSE y la lealtad de los clientes intermediarios, determinando como influye la RSE en la lealtad. Como resultado primero verificaron la confianza con el coeficiente alfa de Cronbach y concluyeron que la RSE ejerce una influencia mayor sobre la lealtad actitudinal afectiva y la comportamental. (Niño de Guzmán M., Rodríguez G., y Rodríguez M., 2016).

Una evidencia de lo mencionado anteriormente son las instituciones del sector financiero en Ibagué, las cuales fueron analizadas por la Facultad de Economía y Administración de la Universidad del Tolima a través de un estudio mixto que se realizó, abordando aspectos relacionados con la investigación cuantitativa y cualitativa. La recolección de datos se realizó a través de un cuestionario estructurado con escala tipo Likert de 36 ítems, recogiendo aspectos relacionados con trabajadores, consumidores y clientes, comunidad, locales, proveedores, medio ambiente y gobierno corporativo. Dentro de los instrumentos utilizados fue el coeficiente de correlación de Pearson para confirmar la validez del contenido, junto con una muestra piloto a 5 bancos. El índice de confiabilidad se realizó a través de la técnica factorial por componentes principales y del cálculo del coeficiente alfa de Cronbach. La investigación concluyo evaluaciones bajas y moderadas en las diferentes dimensiones de la RSE, en donde todavía falta mucha conciencia por parte de las entidades financieras para entender que además de generar ganancias se debe ofrecer bienestar a la sociedad. (Rubio Guerrero, 2016) 
Para evitar lo anterior, se enmarcó la Responsabilidad Empresarial en diferentes normas, las cuales se clasifican en 3 categorías, normas generales que incluyen la gestión de la responsabilidad corporativa, normas de recomendaciones sobre la ética de las empresas y la última categoría son los estándares que guían sobre como evidencias la responsabilidad corporativa. Las normas a las que se hace referencia son SA8000, G3, AA1000, Modelo EFQM de RC, EFR1000, Global Compact, ISO 26000. Esta última fue el resultado de diversas conferencias que se realizaron en diversos países, como lo son Salvador de Bahía y Boangkok en el 2005, Lisboa en el 2006, Sídney y Viena en el 2007, Santiago de Chile (2008), Quebec (2009) y Copenhague (2010). Todas estas reuniones acogieron a más de 450 expertos, 210 observadores todos ellos de 99 países que dieron como resultado en el 1 de noviembre de 2014 la Norma ISO 26000 de responsabilidad social a nivel mundial. La necesidad de este documento surgió porque no se tenía una norma la cual se pudiera aplicar a todo tipo de empresa dejando a muchas sin una guía para la adopción de la Responsabilidad Empresarial. (Andía Valencia, 2015).

En el tercero y último presenta los Conceptos y Evolución (Aportes) a los temas que se encuentran en el trabajo, en este último se encuentra temas como los impactos de las prácticas de la RS, la investigación apunta al valor compartido creado por dicha responsabilidad social corporativa (RSE): estas acciones corporativas no solo ayudan a hacer del mundo un lugar mejor, sino que también generan reacciones positivas de los consumidores, ayudando a construir o pulir la reputación de una empresa". (Hildebrandt, 2017).

Lo que se encontró en la revisión bibliográfica, dio un camino por donde se podía abordad el planteamiento del problema y da un resultado a lo que se propone con la investigación.

\section{DISCUSIÓN Y PROPUESTA}

La responsabilidad social en las empresas se ha desarrollado rápidamente en los últimos años a raíz de la globalización, la evolución de los medios de información, las economías emergentes y las redes sociales. Para esta investigación se realizaron encuestas, entrevistas y dinámicas grupales que permitieron observar que en la visión y misión hacía falta incluir conceptos como el entorno, valores y comunidad; los cuales son elementos muy fundamentales de la RSE. Finalmente, se creó un plan de acción en el que se inicia evaluando hasta qué punto las iniciativas actuales involucran las siguientes variables: Medio ambiente, Público interno, Comunidad, Valores de transparencia 
y finalmente Gobierno y sociedad. Luego, en un Diagrama de Ishikawa, a través de las causas y subcausas, se evidencian el nivel en el que la organización integra la RSE y cómo puede convertirla en una estrategia de la organización danto así valor a la empresa y a sus grupos de interés. (Rodríguez González, 2018)

En la primera etapa de la presente investigación se analizaron varias referencia bibliográficas con el fin de conocer más a fondo sobre el tema, identificar los avances nacionales e internacionales y poder contar con las bases suficientes para incursionar en la segunda etapa y finalmente generar el modelo diagnostico que pueda, no solo evidenciar que están haciendo las mipymes del sector del reciclaje del Barrio Toberín en cuanto a la Responsabilidad Social Empresarial, sino también indicar algunas recomendaciones de cómo mejorar o como iniciar un proceso de implementación de este tema.

Durante la segunda parte, se desarrollaría el modelo diagnóstico que evaluará factores de la Responsabilidad Social Empresarial como el cuidado del medio ambiente, la protección de los Derechos Humanos, el cumplimiento de los derechos laborales y las iniciativas de anticorrupción que tenga la organización. Se pretenden elaborar sentencias fáciles de comprender con respuestas cerradas de si o no que el director de la empresa, empleador o encargado pueda responder desde su computadora o su celular. La aplicación irá evaluando las respuestas para al final indicarle el porcentaje de cumplimiento en cada uno de los factores analizados y le dará recomendaciones para mejorar los factores con menor porcentaje.

El proyecto en su totalidad se desarrollará guiado por la siguiente figura: 


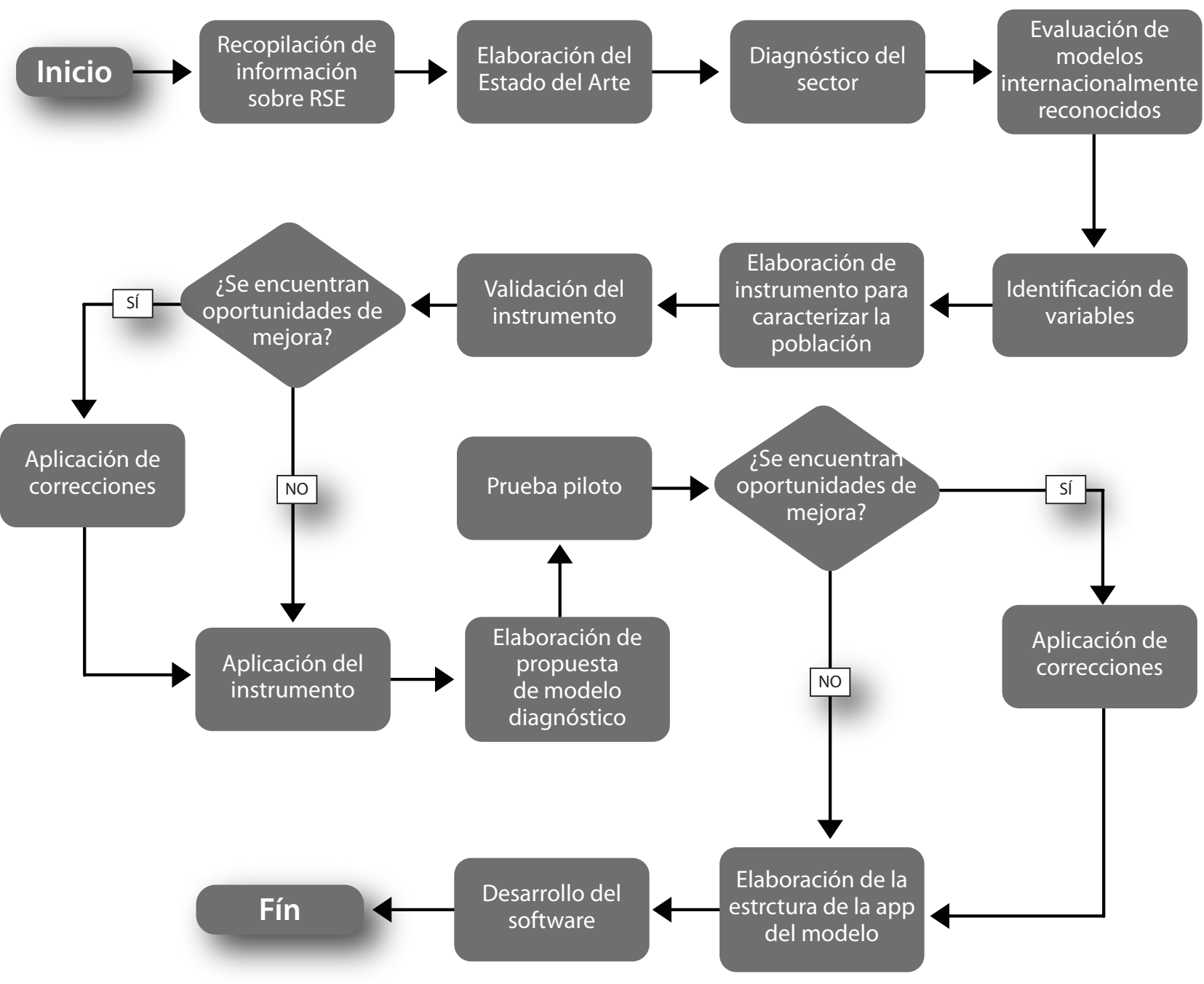

Figura 1. Flujograma del proyecto.

Fuente: elaboración propia 


\section{CONCLUSIONES}

Luego de culminar esta primera etapa de la investigación, se evidencia una necesidad de fortalecer la implementación de la Responsabilidad Social Empresarial en las organizaciones de diferentes sectores en Colombia. Al mismo tiempo, los beneficios obtenidos por dicha implementación no son claros para los miembros de las empresas y por ende es muy bajo el interés por invertir en este tema tal como se observó a través de varios estudios de casos realizados durante los últimos años.

Por lo anterior descrito en el documento se puede continuar con la propuesta de esta investigación, debido a que se tiene el soporte teórico suficiente tanto nacional o internacional para hacer un aporte en la segunda etapa a las mipymes del sector del reciclaje que se encuentran en el barrio Toberín, puesta que es un sector empresarial de fácil acceso sin que esto represente que el modelo diagnóstico no pueda ser utilizado en otros sectores.

\section{REFERENCIAS}

Codina Jiménez, A. (2010). El arte de la ejecución de la estrategia. (pp. 213-224). El arte de la ejecución de la estrategia. (pp. 213-224). Recuperado de http://www.redalyc.org/pdf/1513/151316944004.pdf

Fernández Fernández, J. L., y Bajo Sanjuán, A. (2012). La teoría del Stakeholder o de los grupos de interés, pieza clave de la RSE, del éxito empresarial y de la sostenibilidad. Recuperado de http://adresearch. esic.edu/files/2012/06/aDR6-07-teoria_stakeholder.pdf

Grandolí Sandemetrio, A. J. (2014/2015). Análisis de la Teoría de los sentimientos morales y La riqueza. O Recuperado de http://neurofilosofia.com/wp-content/uploads/2016/11/TFG-ADAM-SMITH-ysu-relaci\%C3\%B3n-con-la-RSE.-Arturo-Gradoli.-06-2015.pdf

Newman, G. (2006). El razonamiento inductivo y deductivo dentro del proceso investigativo en ciencias experimentales y sociales. Obtenido del razonamiento inductivo y deductivo dentro del proceso investigativo en ciencias experimentales y sociales. 
https://doi.org/10.22490/25392786.4042

Organización Internacional del Trabajo OIT. (1996-2019). Recuperado de https://www.ilo.org/global/ about-the-ilo/history/lang--es/index.htm.

Paneque, R. (1998). Metodología De La Investigación. Obtenido de Metodología De La Investigación.

Rodriguez Daponte, R., Dopico Parada, A., y González Vázquez, E. (s.f.). La responsabilidad social empresarial. Un Acercamiento a la realidad empresarial de Galicia. Recuperado de https://dialnet. unirioja.es/descarga/articulo/2751754.pdf

Scaramussa, S. A., Reisdorfer, V. K., y Ribeiro, A. A. (2010). La Contribución Del Balanced Scorecard Como Instrumento De Gestión Estratégica En El Apoyo De La Gerencia. Recuperado de http://www. redalyc.org/pdf/3579/357935475003.pdf

Acevedo Guerrero, J. A., Zárate Rueda, R., y Garzón Ruiz, W. F. (2013). Estatus jurídico de la Responsabilidad Social Empresarial (RSE) en Colombia.

Del Castillo, C. C. (2008). Responsabilidad Social Empresarial. Facultad de Economía y Negocios Universidad de Chile.

Freeman, R. E. (2008). Strategic Management : A Stakeholder Approach. Estados Unidos: Cambridge India.

Hildebrandt, D. (2017). Estudio de impacto de las práctivas de RS. EEUU.

Pastrana, N. (2014). Corporate Social Responsibility: Perceptions and practices among SMEs in Colombia. Public Relations Review, 14-24.

Plasencia Soler, J. A., Marrera Delgado, F., Bajo Sanjuán, A. M., \& Nicado García, M. (Mar de 2018). Modelos para evaluar la sostenibilidad de las organizaciones, 34(146), 63-73. Recuperado de http:// www.scielo.org.co/pdf/eg/v34n146/0123-5923-eg-34-146-00063.pdf

Playán, I. F. (2010). Responsabilidad social empresarial en américa latina: Un panorama general. Administracion y Organizaciones, 57-73. 
Reyno Momberg, M. (2007). Responsabilidad Social Empresarial como ventaja competitiva.

Smith, A. (2013). Teoría de los Sentimiento Morales. España: Alianza Editorial.

Vives, A. (2014). Guías para la Responsabilidad Social en las PyMEs: Efectividad de herramientas de autoevaluación. Recuperado de https://gcg.universia.net/article/viewFile/468/594 